\title{
LncRNA KCNQ1OT1 controls cell proliferation, differentiation and apoptosis by sponging miR-326 to regulate c-Myc expression in acute myeloid leukemia
}

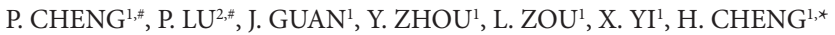 \\ ${ }^{1}$ Department of Hematology, Wuhan No. 1 Hospital, Wuhan, Hubei, China; ${ }^{2}$ Department of Anesthesiology, Tongji Hospital, Tongji Medical \\ College, Huazhong University of Science and Technology, Wuhan, Hubei, China \\ ${ }^{*}$ Correspondence: lijjnl23@163.com \\ ${ }^{*}$ Contributed equally to this work.
}

Received December 15, 2018 / Accepted June 11, 20197

\begin{abstract}
Long noncoding RNAs (lncRNAs) have been reported to play essential roles in development and treatment of acute myeloid leukemia (AML). However, the role of lncRNA potassium voltage-gated channel subfamily Q member 1 overlapping transcript 1 (KCNQ1OT1) in AML progression and its mechanism remain largely unknown. The expressions of KCNQ1OT1, microRNA-326 (miR-326) and c-Myc were measured by quantitative real-time polymerase chain reaction and western blot, respectively. Phorbol myristate acetate (PMA) was used for cell differentiation. Cell proliferation, apoptosis and differentiation were measured by MTT assay, flow cytometry and qRT-PCR, respectively. The interaction between miR-326 and KCNQ1OT1 or c-Myc was explored by luciferase activity, RNA immunoprecipitation or RNA pull-down assay. We found that the expression of KCNQ1OT1 was enhanced in AML samples compared with control. KCNQ1OT1 knockdown inhibited cell proliferation but promoted apoptosis and cell differentiation. KCNQ1OT1 was a decoy of miR-326 and c-Myc was a target of miR-326. KCNQ1OT1 regulated AML cell proliferation, apoptosis and differentiation by sponging miR-326. Moreover, overexpression of miR-326 suppressed proliferation but promoted apoptosis and PMA-induced differentiation by targeting c-Myc in AML cells. Besides, c-Myc protein level was suppressed by KCNQ1OT1 interference and rescued by miR-326 abrogation. Our data showed that KCNQ1OT1 regulates proliferation, differentiation and apoptosis in AML cells by acting as a competing endogenous RNA (ceRNA) for miR-326 to regulate c-Myc, providing a novel avenue for AML treatment.
\end{abstract}

Key words: acute myeloid leukemia, KCNQ1OT1, miR-326, c-Myc, proliferation, differentiation

Acute myeloid leukemia (AML) is a bone marrow malignancy with rising incidence worldwide [1]. AML malignancy is characterized by uncontrolled proliferation, impaired apoptosis and interferes with programmed differentiation in leukemic cells [2]. Hence, it is necessary to explore novel avenue for treatment of AML through suppressing cell growth and overcoming differentiation arrest.

Long noncoding RNAs (lncRNAs) are confined to transcripts lacking significant ORFs with more than 200 nt in length, which play important roles in tumorigenesis in progression of disorders, including AML [3]. Moreover, lncRNAs have been reported to be associated with prognosis of AML by functioning as competing endogenous RNAs (ceRNAs) [4]. Previous studies have reported that lncRNAs are implicated in AML progression by regulating cell processes, including proliferation, apoptosis, migration, invasion and differentiation [5-7]. As for lncRNA potas- sium voltage-gated channel subfamily Q member 1 overlapping transcript 1 (KCNQ1OT1), it has been reported to act as a carcinogenic lncRNA in multiple cancers by promoting cancer cell proliferation, migration, invasion and epithelialmesenchymal transition [8-10]. Furthermore, former work suggested that KCNQ1OT1 could promote osteoblast differentiation [11]. Jia et al. revealed that KCNQ1OT1 is highly expressed in AML and might be associated with prognosis and therapeutics of AML patients [12]. However, little is known about the roles of KCNQ1OT1 and its mechanism in AML.

miRNAs are a class of small noncoding RNAs with 19-22 nucleotides, which play essential roles in diagnosis, prognosis and therapeutics in AML [13]. Accruing reports suggested miRNA-326 as a tumor suppressor to inhibit proliferation, invasion, cell cycle progression and to promote apoptosis in many cancers, such as prostate cancer, osteosarcoma and hepatocellular carcinoma [14-16]. Moreover, miR-326 has 
been suggested to be dysregulated in AML samples [17]. c-Myc is regarded as an oncogene and has an important role in development of AML [18]. Moreover, c-Myc has been reported to be associated with cell growth, differentiation and drug resistance in AML by varying pathways [7, 19]. Notably, bioinformatics analysis displays the potential binding sites of miR-326 and c-Myc or KCNQ1OT1. Thus, we hypothesized that KCNQ1OT1 might act as a ceRNA for miR-326 to regulate c-Myc in AML. In this study, we investigated the effect of KCNQ1OT1 on cell differentiation, proliferation and apoptosis in vitro and explored the potential ceRNA regulatory network of KCNQ1OT1/miR-326/c-Myc.

\section{Materials and methods}

Samples. The peripheral blood samples from 41 AML patients and 20 healthy volunteers were recruited from Wuhan No.1 Hospital. The peripheral blood mononuclear cells (PBMCs) of each sample were isolated by using FicolHistopaque gradient centrifugation method [20]. All participants have signed the informed consent and this study was approved by the Research Ethics Committee of Wuhan No.1 Hospital. The 5-year survival was analyzed in AML patients according to the expression of KCNQ1OT1.

Cell culture and treatment. The human myeloid leukemia cell lines HL-60 and U937 cells were purchased from American Tissue Culture Collection (Manassas, VA, USA) and cultured in Dulbecco's Modified Eagle Medium (Gibco, Carlsbad, CA, USA) with 10\% fetal bovine serum (Gibco), $100 \mathrm{U} / \mathrm{ml}$ penicillin and $100 \mu \mathrm{g} / \mathrm{ml}$ streptomycin (Invitrogen, Carlsbad, CA, USA) at $37^{\circ} \mathrm{C}$ and $5 \% \mathrm{CO}_{2}$. For cell differentiation, HL-60 and U937 cells were treated with $50 \mathrm{ng} / \mathrm{ml}$ phorbol myristate acetate (PMA; Sigma, MO, USA) dissolved in dimethylsulfoxide (DMSO; Thermo Fisher, DE, USA) for $0,24,48$ or $72 \mathrm{~h}$.

Small interfering RNA (siRNA) against KCNQ1OT1 (si-KCNQ1OT1), scramble, vector, KCNQ1OT1 overexpression vector (KCNQ1OT1), c-Myc overexpression vector (c-Myc), miR-326 mimic (miR-326), negative control (NC), miR-326 inhibitor (anti-miR-326) and miRNA inhibitor NC (anti-NC) were synthesized by Genepharma (Shanghai, China). Cell transfection was performed in HL-60 and U937 cells by using Lipofectamine 2000 (Invitrogen) following the manufacturer's instructions. After $24 \mathrm{~h}$ of the transfection, cells were collected for further study.

Quantitative real-time polymerase chain reaction (qRT-PCR). Total RNA was isolated from cells with TRIzol reagent (Invitrogen) and reverse transcribed using TransScript miRNA First stand cDNA Synthesis SuperMix (TransGen Biotech, Beijing, China) according to the manufacturer's instructions. qRT-PCR was conducted by using SYBR green (Vazyme Biotech, Nanjing, China) and each sample was prepared in triplicate. The relative levels of RNAs were measured with U6 small RNA or GAPDH as internal control using $2^{-\Delta \Delta C t}$ method [21]. The primers were listed as follows:
miR-326 (Forward, 5'-CCTCTGGGCCCTTCCTCCAG-3'; Reverse, 5'-GCGAGCACAGAATTAATACGAC-3'), U6 (Forward, 5'-CTCGCTTCGGCAGCACATATACT-3'; Reverse, 5'-ACGCTTCACGAATTTGCGTGTC-3'), KCNQ1OT1 (Forward, 5'-GCACTCTGGGTCCTGTTCTC-3'; Reverse, 5'-CACTTCCCTGCCTCCTACAC-3'), cluster of differentiation 14 (CD14) (Forward, 5'-CTCTGTCCTTAAAGCGGCTTAC-3'; Reverse, 5'-GTTGCGGAGGTTCAAGA TGTT-3'), CD11b (Forward, 5'-TCCAAAACACGGGGACCTATC-3'; Reverse, 5'-TCCTCGAACACGACCACCT-3'), c-Myc (Forward, 5'-GGCTCCTGGCAAAAGGTCA-3'; Reverse, 5'-CTGCGTAGTTGTGCTGATGT-3'), GAPDH (Forward, 5'-GGCAGCAGCAAGCATT CCT-3'; Reverse, 5'-GCCCAACACCCCCAGTCA-3').

Cell proliferation. 3-(4,5-dimethyl-2-thiazolyl)-2,5diphenyl-2-H-tetrazolium bromide (MTT) assay was conducted to analyze cell proliferation. HL-60 and U937 cells were seeded into 96-well plates at a density of 5,000 cells per well and each sample was prepared in triplicate. After the culture for 24,48 or $72 \mathrm{~h}$, cells were incubated with $0.5 \mathrm{mg} / \mathrm{ml}$ MTT solution (Thermo Fisher) for another $4 \mathrm{~h}$. Subsequently, $100 \mu \mathrm{l}$ of DMSO was added to each well for $10 \mathrm{~min}$ and the absorbance was measured at $490 \mathrm{~nm}$ using a microplate reader (Bio-Rad, CA, USA).

Cell apoptosis. Cell apoptosis was analyzed by using Annexin V-fluorescein isothiocyanate (FITC)/propidium iodide (PI) apoptosis detection kit (Yeasen, Shanghai, China) via flow cytometry. After the culture for $72 \mathrm{~h}, \mathrm{HL}-60$ and U937 cells were washed with PBS and resuspended in binding buffer, followed by stained with $5 \mu \mathrm{l}$ Annexin V-FITC and $10 \mu \mathrm{l}$ PI for $10 \mathrm{~min}$ in the dark at room temperature. The stained cells were analyzed by using a flow cytometer (Becton Dickinson, Franklin Lakes, NJ, USA).

Luciferase activity assay. The putative binding sites of miR-326 and KCNQ1OT1 or c-Myc were predicted by starBase or TargetScan online. The 3' untranslated regions (3'-UTR) sequences of KCNQ1OT1 or c-Myc containing wild-type (wt) or mutant (mut) binding sites of miR-326 were inserted into the pGL3 vectors (Promega, Madison, WI, USA) to synthesize luciferase reporter vectors (KCNQ1OT1wt, KCNQ1OT1-mut, c-Myc-wt or c-Myc-mut). HL-60 and U937 cells were co-transfected with 20 ng luciferase reporter vector, $10 \mathrm{ng}$ control vector and $40 \mathrm{nM}$ miR-326 or miR-NC using Lipofectamine ${ }^{\text {Tw }} 2000$ according to the manufacturer's protocols. After the transfection for $48 \mathrm{~h}$, luciferase activity assay was measured using luciferase assay kit (Promega) according to the manufacturer's instructions.

RNA immunoprecipitation (RIP). RIP assay was performed by using RNA-binding protein immunoprecipitation kit (Millipore, Billerica, MA, USA) according to the manufacturer's protocols. In brief, HL-60 and U937 cells transfected with miR-326 or miR-NC were lysed in RIP buffer containing magnetic beads bound with antibody against Ago 2 or IgG. The enrichment of KCNQ1OT1 or c-Myc immunoprecipitated on beads were detected by qRT-PCR. 
RNA pull-down assay. RNA pull-down analysis was performed in HL-60 and U937 cells by using RNA-Protein Pull-Down Kit (Thermo Fisher). In brief, NC or miR-326 was labeled with biotin and transfected into cells. The cell lysates were incubated with streptavidin agarose beads (Invitrogen) for $2 \mathrm{~h}$, and then eluted with biotin elution buffer. The complex was used for measurement of KCNQ1OT1 level by qRT-PCR.

Western blot. After washed with PBS, HL-60 and U937 cells were lysed by using RIPA lysis buffer (Beyotime Biotech, Shanghai, China). Total proteins were quantified by BCA protein assay kit (Beyotime Biotech) following the centrifugation and then denatured at $100^{\circ} \mathrm{C}$ for $10 \mathrm{~min}$. Equal amounts of proteins were separated on SDS-PAGE gel and then transferred to polyvinylidene difluoride (PVDF) membranes (Millipore). The membranes were treated with $1 \%$ bovine serum albumin (Sigma) for $1 \mathrm{~h}$ at room temperature, and then incubated with primary antibodies overnight at $4^{\circ} \mathrm{C}$ and secondary antibody for $2 \mathrm{~h}$ at room temperature. The antibodies against c-Myc (ab32072, 1:1000 dilution) or GAPDH (ab181602, 1:10000 dilution) and horseradish peroxidase (HRP)-conjugated secondary antibody (ab205718, 1:10000 dilution) were purchased from Abcam (Cambridge, UK). GAPDH was used as loading control and the protein blots were visualized using enhanced chemiluminescence (ECL) chromogenic substrate (Beyotime Biotech).

Statistical analysis. Data were presented as the mean \pm standard deviation (S.D.) from three independent experiments. The statistical differences between groups were analyzed by Student's t-test or one-way analysis of variance (ANOVA) using GraphPad Prism 5 (GraphPad Inc., La Jolla, CA, USA). The overall survival curve of patients was generated by Kaplan-Meier method and analyzed by Log-rank test. A p-value $<0.05$ was regarded as statistically significant.

\section{Results}

KCNQ10T1 expression is enhanced in AML samples. To explore the potential role of KCNQ1OT1 in AML progres- sion, its expression was measured in AML samples. As shown in Figure 1A, the expression of KCNQ1OT1 was abnormally increased in PBMCs from AML patients compared with that in normal group. Moreover, the patients were classified as low expression $(n=26)$ and high expression $(n=15)$ groups according to the mean value of KCNQ1OT1 expression in AML samples. After the follow-up, high expression of KCNQ1OT1 group displayed lower survival rate of patients $(\mathrm{p}=0.005)$ (Figure 1B).

KCNQ1OT1 regulates differentiation, proliferation and apoptosis in AML cells. To investigate the role of KCNQ1OT1 in cell differentiation, AML cells were stimulated with PMA. As shown in Figure 2A and 2B, the expression levels of CD14 and CD11b mRNA were significantly elevated in HL-60 and U937 cells at 24, 48 and $72 \mathrm{~h}$ after the treatment of PMA compared with those in control and DMSO groups, suggesting that PMA effectively induced the cell differentiation. Moreover, the abundance of KCNQ1OT1 was progressively decreased in PMA-treated HL-60 and U937 cells (Figure 2C), indicating that KCNQ1OT1 might be associated with cell differentiation. To explore the effect of KCNQ1OT1 on AML progression, its abundance was effectively knocked down in the two cell lines by using siRNA, which was revealed by qRT-PCR (Figure 2D). The analysis of MTT revealed that knockdown of KCNQ1OT1 significantly reduced proliferation of HL-60 and U937 cells compared with scramble group at $72 \mathrm{~h}$ (Figure 2E). Besides the $72 \mathrm{~h}$ time point, cell apoptosis was notably induced by silencing KCNQ1OT1 in HL-60 and U937 cells compared with that in scramble group (Figure 2F). Meanwhile, the data of qRT-PCR displayed that cell differentiation was promoted by silencing KCNQ1OT1 in the PMA-challenged cells, revealed by the increased CD14 and CD11b mRNA levels at $72 \mathrm{~h}$ (Figures $2 \mathrm{G}$ and $2 \mathrm{H}$ ).

KCNQ1OT1 regulates AML cell differentiation, proliferation and apoptosis by sponging miR-326. Seeing that functional lncRNA was known as ceRNA or miRNA sponge, the miRNAs bound to KCNQ1OT1 were explored by starBase. As described in Figure 3A, the potential

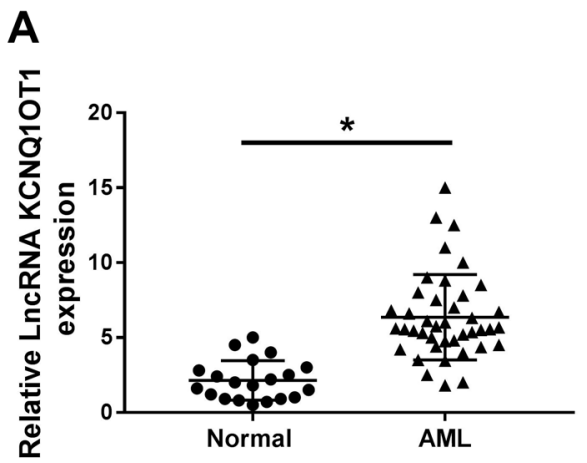

B

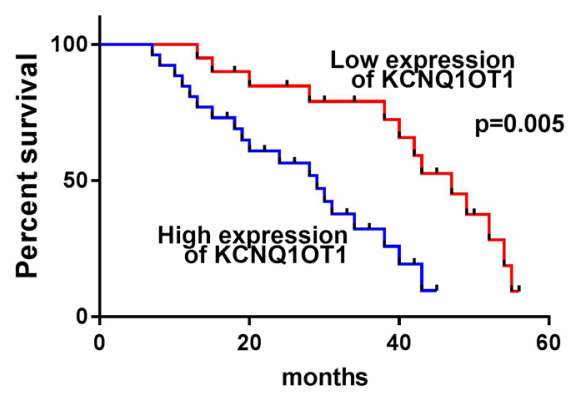

Figure 1. KCNQ1OT1 expression is enhanced in AML patient samples. A) The expression of KCNQ1OT1 was measured in PBMCs cells from AML patients $(n=41)$ or normal controls $(n=20)$ by $q R T-P C R$. B) The survival of AML patients was analyzed according to the expression of KCNQ1OT1. ${ }^{*} \mathbf{p}<0.05$. 
binding sites of miR-326 and 3'-UTR of KCNQ1OT1 were provided, suggesting that KCNQ1OT1 might be a decoy of miR-326. Moreover, the interaction between miR-326 and KCNQ1OT1 was explored by luciferase activity, RIP and RNA pull-down assays. Results showed that overexpression of miR-326 significantly reduced the luciferase activity of HL-60 and U937 cells transfected with KCNQ1OT1-wt, while its efficacy was lost with respect to KCNQ1OT1-mut group (Figure 3B). Moreover, the enrichment of KCNQ1OT1 by Ago2 RIP was obviously enhanced in HL-60 and U937 cells transfected with miR-326 compared with that in NC group (Figure 3C). In addition, biotin-labeled miR-326
A

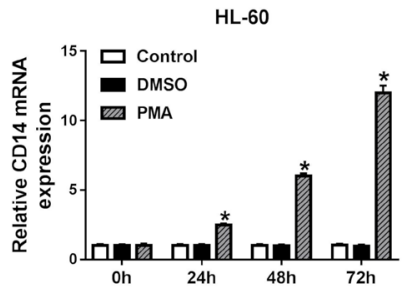

C

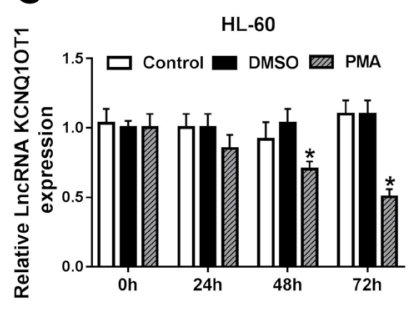

E

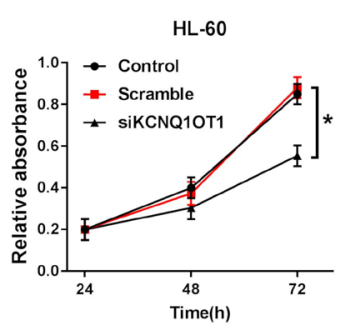

G

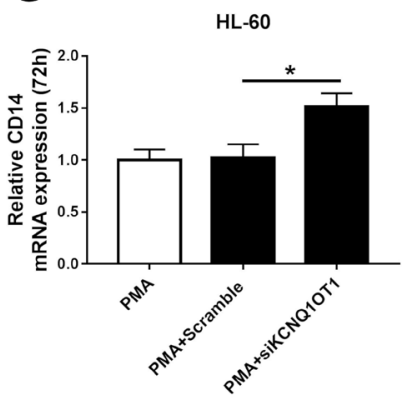

U937
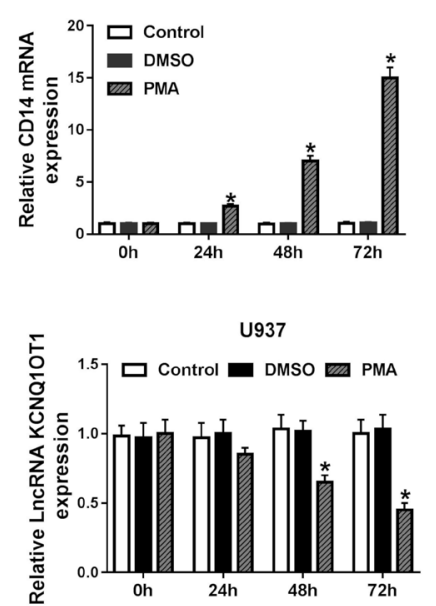

B
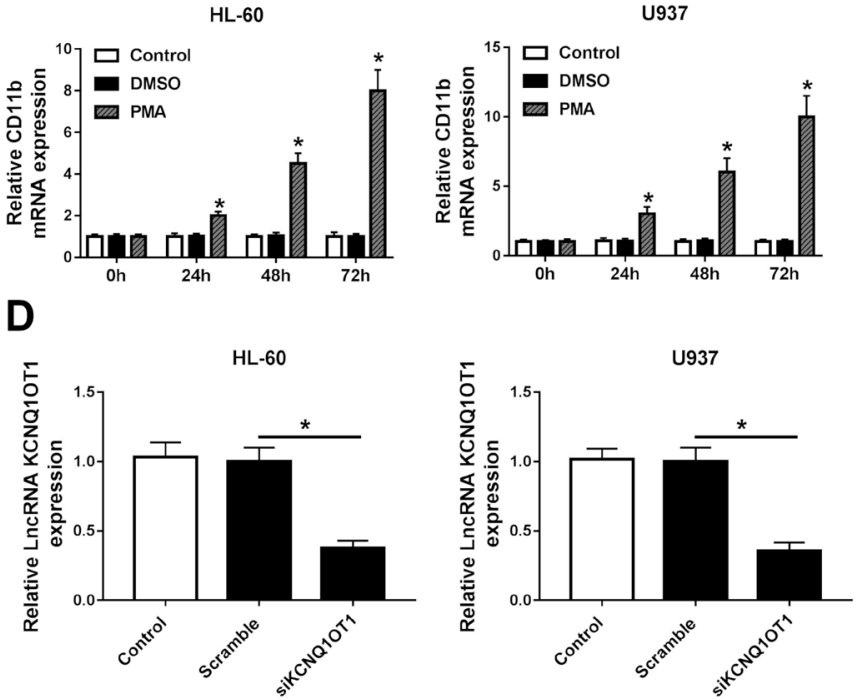

$\mathbf{F}$
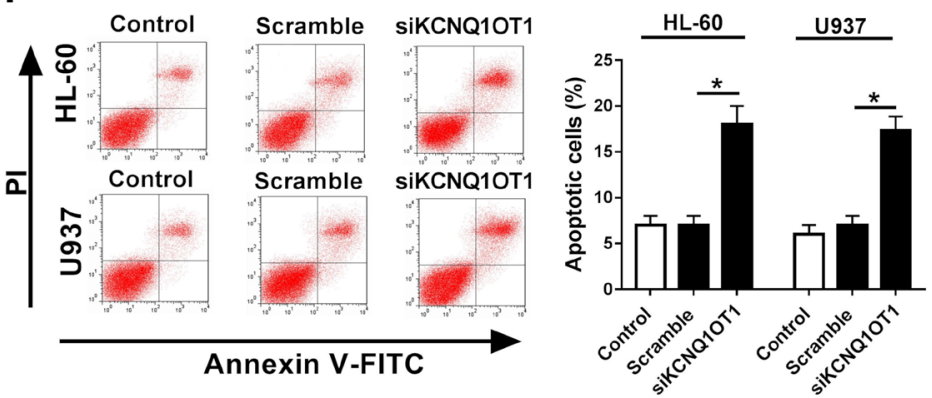

H
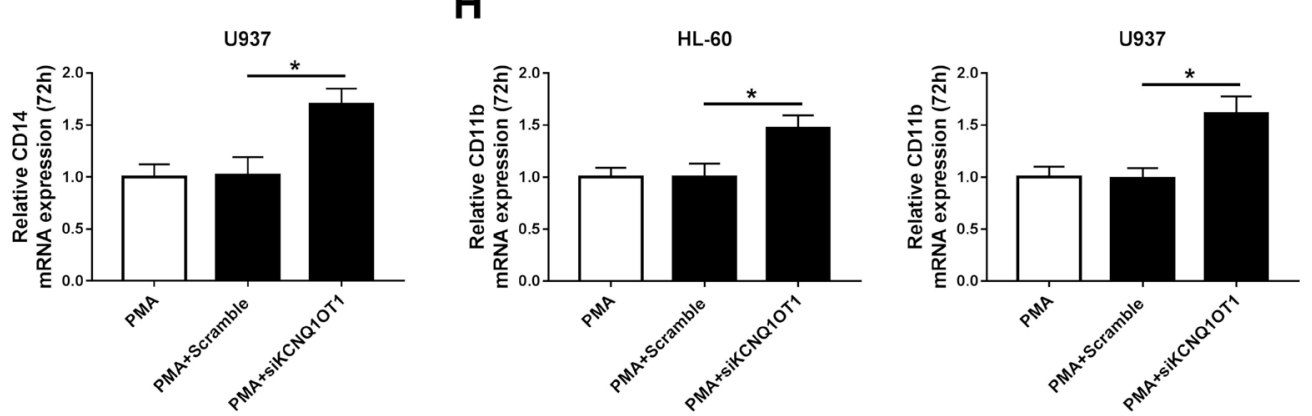

Figure 2. KCNQ1OT1 regulates differentiation, proliferation and apoptosis in AML cells. A and B) The expression levels of CD14 and CD11b mRNA were measured in HL-60 and U937 cells at 0, 24, 48 or $72 \mathrm{~h}$ after treatment of PMA by qRT-PCR. DMSO: negative control group; Control: non-treated group. C) The level of KCNQ1OT1 was detected in HL-60 and U937 cells at $0,24,48$ or $72 \mathrm{~h}$ after treatment of PMA by qRT-PCR. D) The abundance of KCNQ1OT1 was examined in HL-60 and U937 cells transfected with siKCNQ1OT1 or scramble by qRT-PCR. Control: non-transfected group. E) Cell proliferation was measured in HL-60 and U937 cells transfected with siKCNQ1OT1 or scramble at 24, 48 or $72 \mathrm{~h}$ by MTT assay. F) Cell apoptosis was analyzed in HL-60 and U937 cells transfected with siKCNQ1OT1 or scramble at $72 \mathrm{~h}$ by flow cytometry. G and H) The expression levels of CD14 and CD11b mRNA were measured in HL-60 and U937 cells at $72 \mathrm{~h}$ after transfection of siKCNQ1OT1 or scramble and treatment of PMA by qRT-PCR. ${ }^{*} \mathbf{p}<0.05$. 


\section{A}

Target Location chr11:2707204-2707224[-]

miR-326 3'-gaCCTCCTTC - CCGGGTCTCC-5'

\section{KCNQ10T1-mut 5'-gtCCTCTCTCACCGGGTCTCt-3'}

B
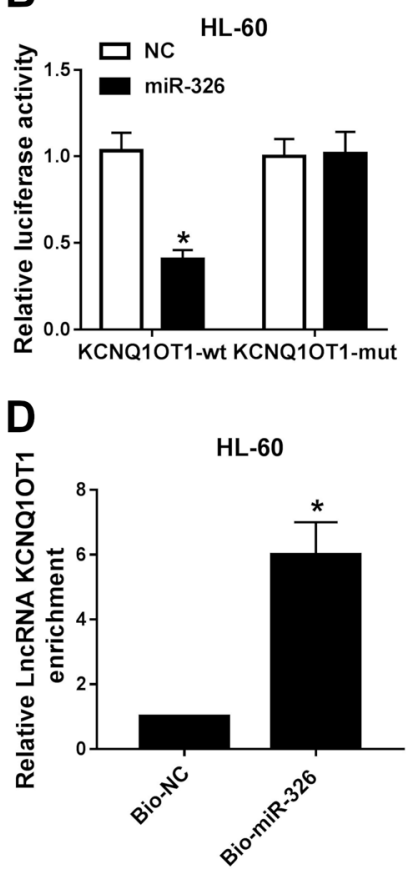
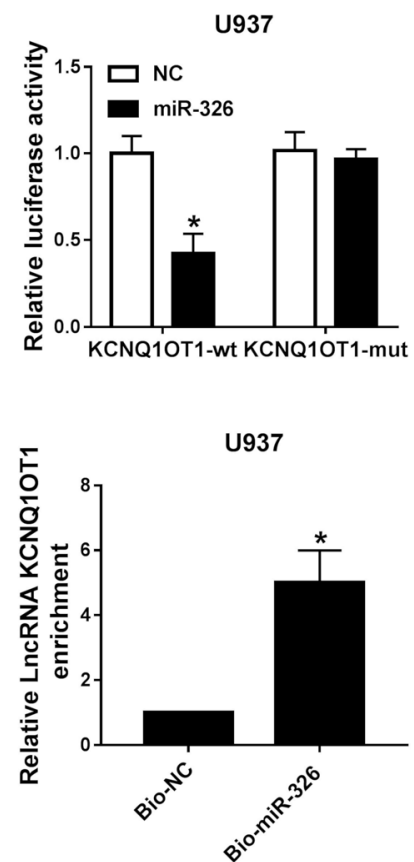
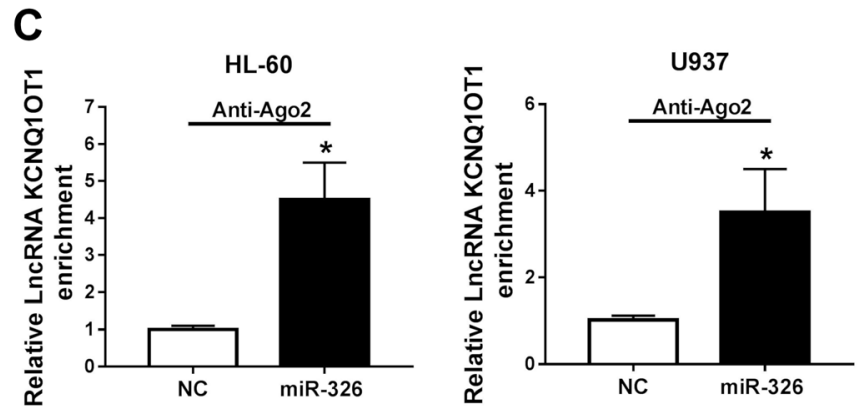

\section{E}

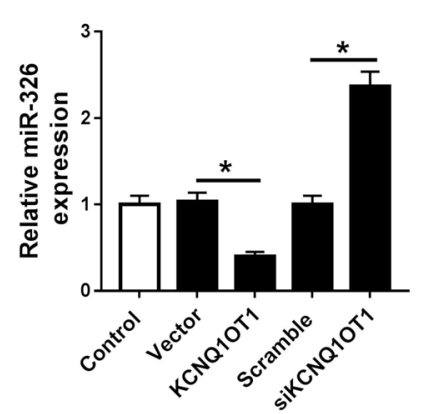

U937

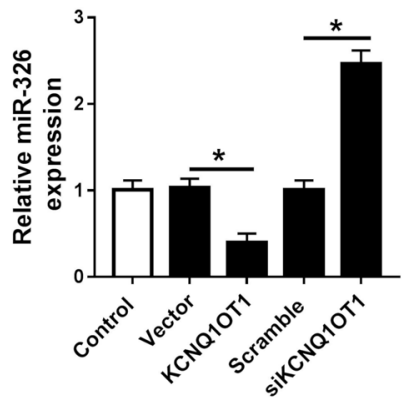

Figure 3. miR-326 is bound to KCNQ1OT1. A) The potential binding sites of miR-326 and KCNQ1OT1 were predicted by starBase. B) Luciferase activity assay was conducted in HL-60 and U937 cells co-transfected with KCNQ1OT1-wt or KCNQ1OT1-mut and miR-326 or NC. C and D) The enrichment of KCNQ1OT1 was measured in HL-60 and U937 cells after Ago2 RIP or RNA pull-down assays. (E) The abundance of miR-326 was detected in HL-60 and U937 cells transfected with vector, KCNQ1OT1, scramble or siKCNQ1OT1 by qRT-PCR. Control: non-transfected group. ${ }^{\star}$ p<0.05.

group displayed higher abundance of KCNQ1OT1 in HL-60 and U937 cells than bio-NC group (Figure 3D). Besides, the expression of miR-326 was evidently decreased by overexpression of KCNQ1OT1 and increased by interference of KCNQ1OT1 in HL-60 and U937 cells (Figure 3E). These findings indicated miR-326 as a target of KCNQ1OT1. As a result, the expression of miR-326 was greatly downregulated in AML samples compared with that in normal (Figure 4A). Furthermore, its level was progressively increased in HL-60 and U937 cells after the treatment of PMA in a time dependent manner, suggesting that miR-326 was involved in cell differentiation (Figure 4B). To investigate the role of miR-326 in AML progression, cells were transfected with miR-326 or NC. After the transfection, miR-326 abundance was significantly elevated in HL-60 and U937 cells transfected with miR-326 compared with that in NC and control group (Figure 4C). In addition, overexpression of miR-326 signifi- cantly limited proliferation but promoted cell apoptosis and PMA-induced differentiation in HL-60 and U937 cells compared with NC group (Figures 4D-4G).

To investigate whether miR-326 was required for KCNQ1OT1-mediated AML progression, AML cells were transfected with scramble, siKCNQ1OT1, siKCNQ1OT1 and anti-NC or anti-miR-326. As a result, the abundance of miR-326 was markedly increased by KCNQ1OT1 knockdown in HL-60 and U937 cells, which was weakened by miR-326 deficiency (Figure 5A). Moreover, downregulation of miR-326 reversed the effect of KCNQ1OT1 silence on proliferation, apoptosis and PMA-induced differentiation in HL-60 and U937 cells at $72 \mathrm{~h}$ (Figures 5B-5E).

miR-326 regulates AML cell differentiation, proliferation and apoptosis by targeting c-Myc. To further explore the mechanism in AML progression, potential target of miR-326 was explored by TargetScan. The putative binding 
A

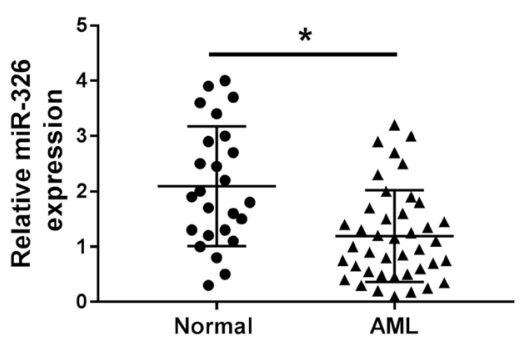

B

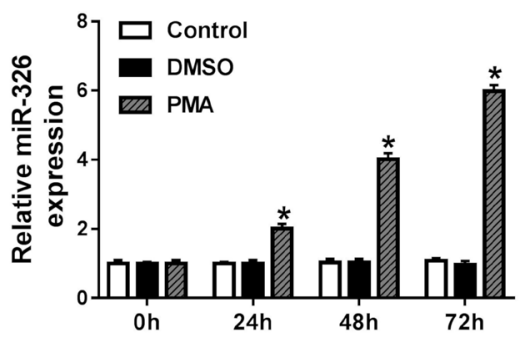

U937
D

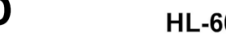

U937

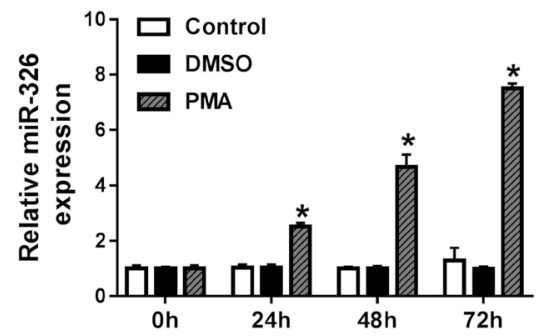

C

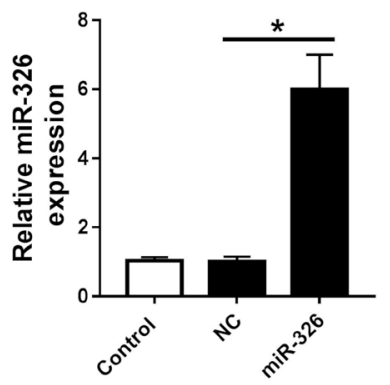

E

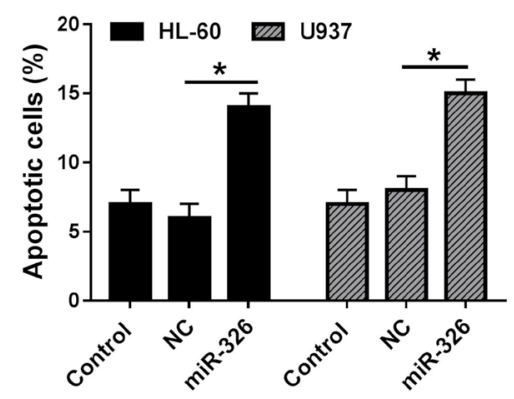

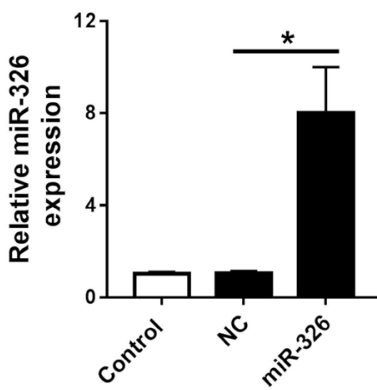

$\mathbf{F}$

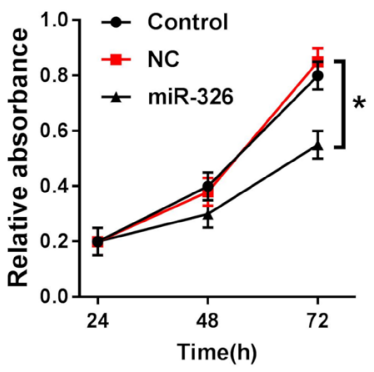

U937

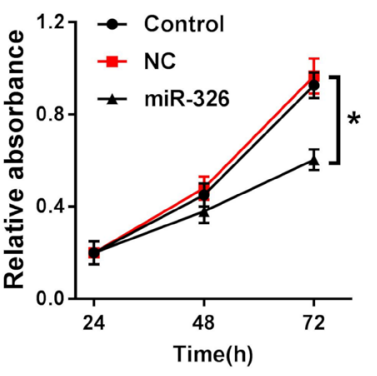

G
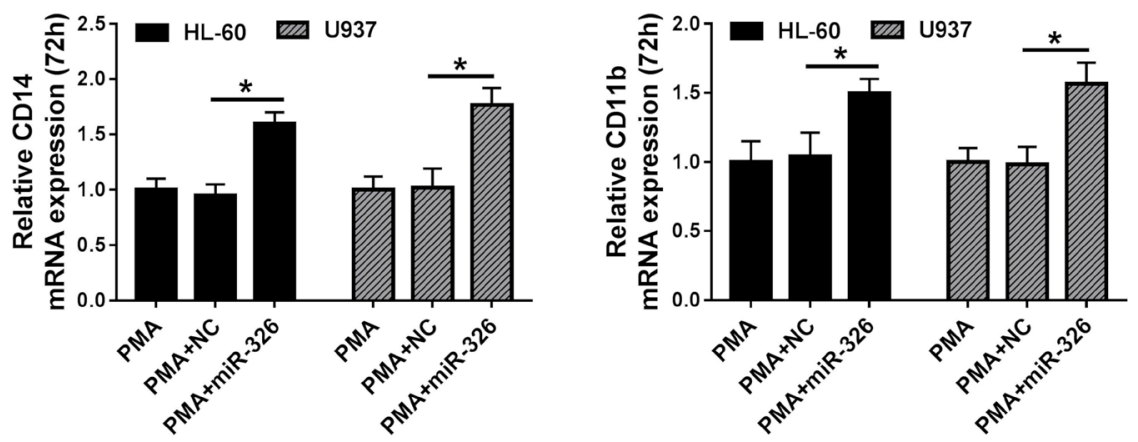

Figure 4. miR-326 regulates differentiation, proliferation and apoptosis in AML cells. A) The expression of miR-326 was measured in PBMCs cells from AML patients or normal controls by qRT-PCR. B) The level of miR-326 was detected in HL-60 and U937 cells at $0,24,48$ or $72 \mathrm{~h}$ after treatment of PMA by qRT-PCR. C) The abundance of miR-326 was examined in HL-60 and U937 cells transfected with miR-326 or NC by qRT-PCR. Cell proliferation (D), apoptosis (E), CD14 and CD11b levels (F and G) were measured in HL-60 and U937 cells transfected with miR-326 or NC at 72 h by MTT assay, flow cytometry or qRT-PCR, respectively. Control: non-treated or non-transfected group. ${ }^{*} \mathrm{p}<0.05$.

sites of miR-326 and c-Myc were shown in Figure 6A, and we constructed the wt or mut luciferase reporter vector. Overexpression of miR-326 resulted in obvious loss of luciferase activity in HL-60 and U937 cells transfected with c-Mycwt, whereas it failed to show efficacy in c-Myc-mut group (Figure 6B). Moreover, accumulation of miR-326 led to great increase of enrichment of c-Myc in HL-60 and U937 cells, while IgG showed little efficacy of enrichment (Figure 6C). Additionally, the expression of c-Myc protein was effectively decreased by overexpression of miR-326 and increased by knockdown of miR-326 in HL-60 and U937 cells (Figure 6D). These data uncovered that c-Myc is a target of miR-326 in AML cells.
As a result, the mRNA level of c-Myc was exceptionally elevated in AML samples compared with that in normal group (Figure 7A). To evaluate whether miR-326-addressed AML progression was mediated by c-Myc, AML cells were transfected with NC, miR-326, miR-326 and vector or c-Myc. After the transfection, western blot assay demonstrated that miR-326 overexpression reduced c-Myc protein level in HL-60 and U937 cells and this event was restored by introduction of c-Myc overexpression vector (Figure 7B). Moreover, restoration of c-Myc weakened miR-326-mediated proliferation suppression and promotion of apoptosis production and PMA-induced differentiation in HL-60 and U937 cells (Figures 7C-7F). 
A

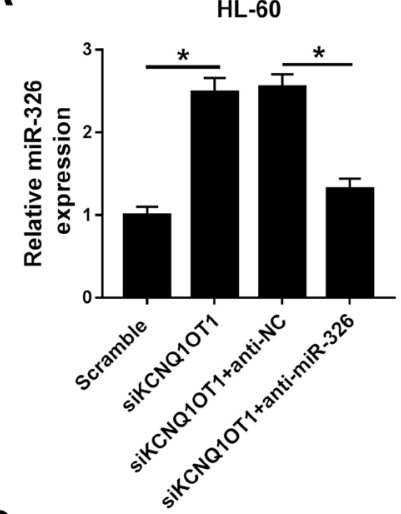

C

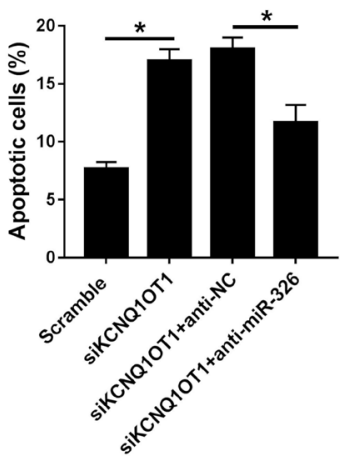

U937

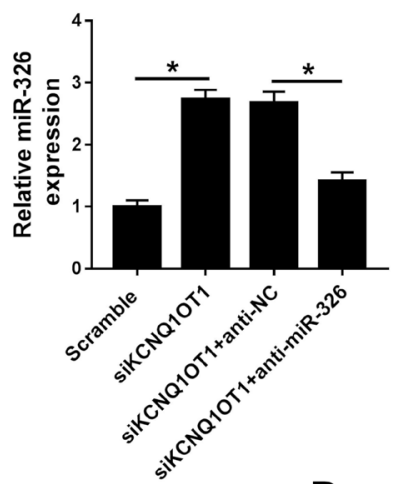

U937

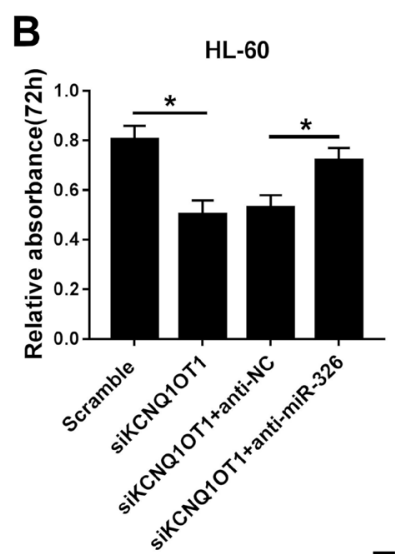

$\mathbf{E}$
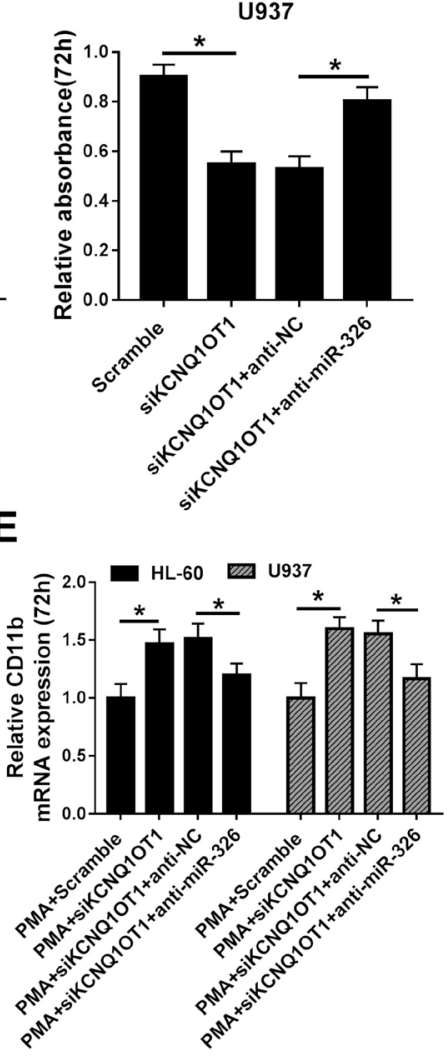

Figure 5. Inhibition of miR-326 reverses the regulatory effect of KCNQ1OT1 knockdown on proliferation, apoptosis and differentiation in AML cells. A) The expression of miR-326 was measured in HL-60 and U937 cells transfected with scramble, siKCNQ1OT1, siKCNQ1OT1 and anti-miR-326 or anti-NC by qRT-PCR. Cell proliferation (B), apoptosis (C), CD14 and CD11b mRNA levels (D and E) were analyzed in HL-60 and U937 cells transfected with scramble, siKCNQ1OT1, siKCNQ1OT1 and anti-miR-326 or anti-NC at $72 \mathrm{~h}$ by MTT assay, flow cytometry or $\mathrm{qRT}-\mathrm{PCR}$, respectively. ${ }^{*} \mathrm{p}<0.05$.

A

$\begin{array}{lrl}\text { c-Myc-wt } & \text { 3' UTR 5' ...GGUUAUCUCGCAAACCCCAGAGG...3' } \\ \text { miR-326 } & \text { 3' GACCUCCUUCCCGGGUCUCC }\end{array}$

c-Myc-mut 3' UTR 5' ...GGUUAUCUCGCAAACGGGUCUCC...3'

C

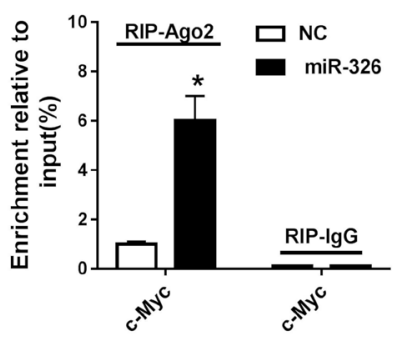

B

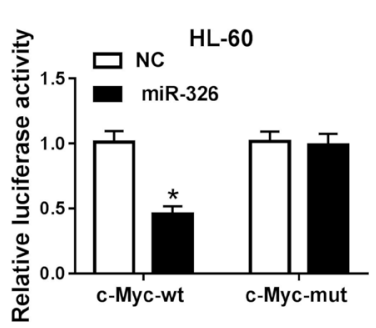

U937

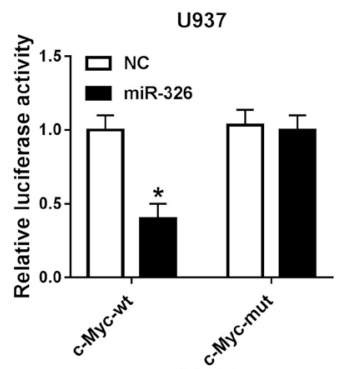

D

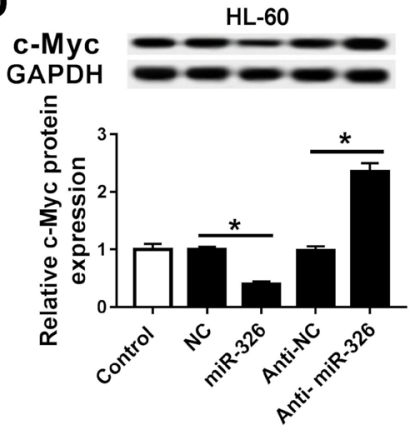

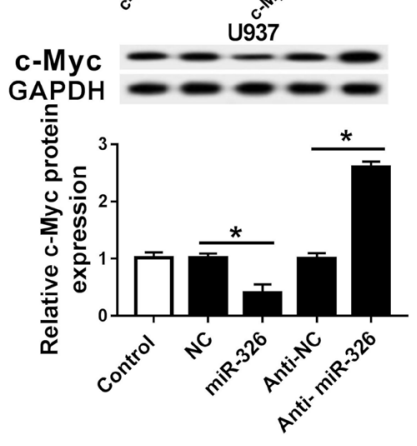

Figure 6. c-Myc is a target of miR-326. A) The putative binding sites of miR-326 and c-Myc was explored by TargetScan. B) Luciferase activity assay was performed in HL-60 and U937 cells co-transfected with c-Myc-wt or c-Myc-mut and miR-326 or NC. C) The enrichment of c-Myc was measured in HL-60 and U937 cells transfected with miR-326 or NC after Ago2 RIP assay. D) The expression of c-Myc protein was detected in HL-60 and U937 cells transfected with miR-326, NC, anti-miR-326 or anti-NC by western blot. ${ }^{*} \mathbf{p}<0.05$. 
A

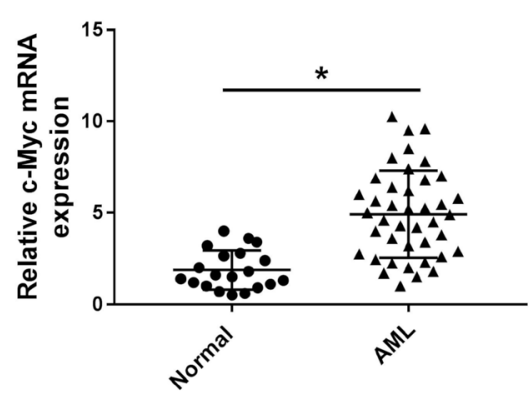

C

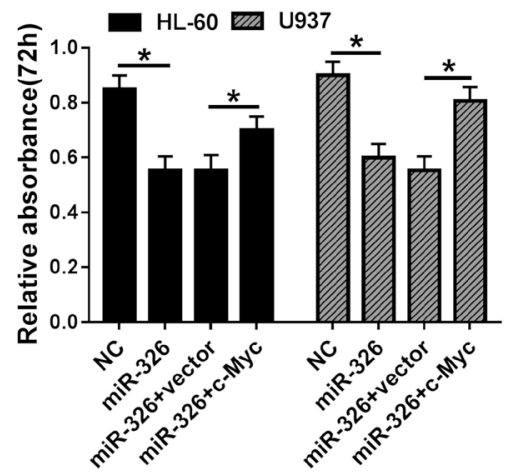

$\mathbf{F}$

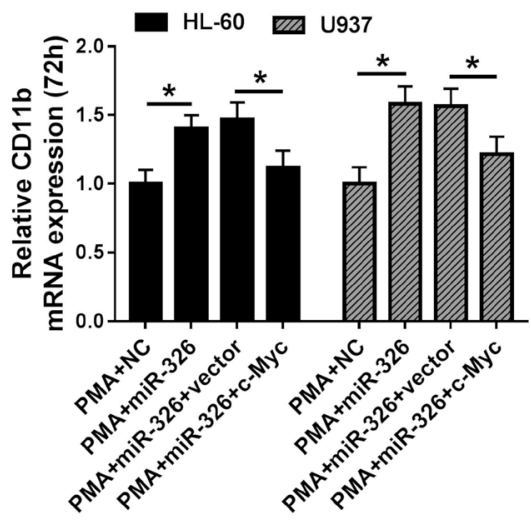

B

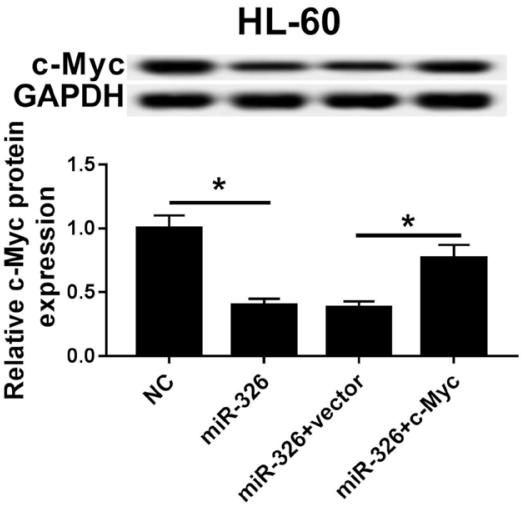

D

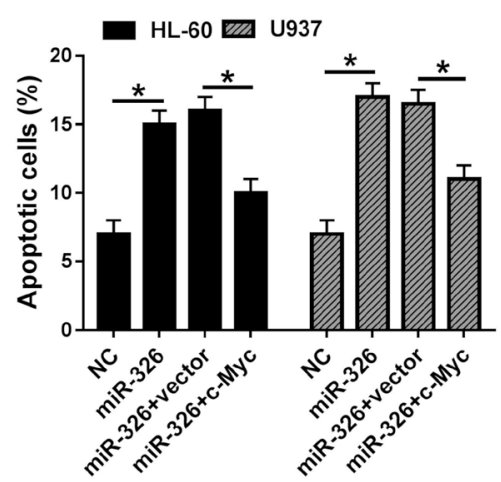

E

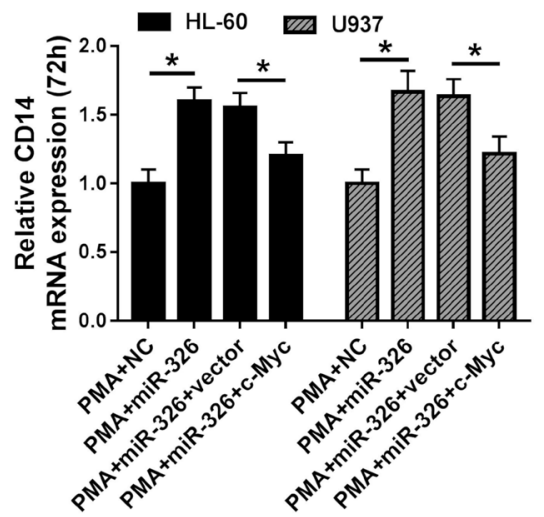

Figure 7. Restoration of c-Myc attenuates miR-326-mediated progression in AML cells. A) The expression of c-Myc mRNA was measured in PBMCs cells from AML patients or normal controls by $\mathrm{qRT}-\mathrm{PCR}$. B) The protein level of c-Myc was detected in HL-60 and U937 cells transfected with NC, miR-326, miR-326 and vector or c-Myc by western blot. Cell proliferation (C), apoptosis (D), CD14 and CD11b mRNA levels (E and F) were examined in HL-60 and U937 cells transfected with NC, miR-326, miR-326 and vector or c-Myc at $72 \mathrm{~h}$ by MTT assay, flow cytometry or qRT-PCR, respectively. ${ }^{*} \mathrm{p}<0.05$.

KCNQ1OT1 regulates c-Myc expression by competitively sponging miR-326 in AML cells. To further elucidate the ceRNA-based mechanism in AML progression, the interaction between c-Myc and KCNQ1OT1 was explored in HL-60 and U937 cells by transfection of scramble, siKCNQ1OT1, siKCNQ1OT1 and anti-NC or anti-miR326. After the transfection of $24 \mathrm{~h}$, western blot results exhibited that knockdown of KCNQ1OT1 significantly decreased c-Myc protein expression in HL-60 and U937 cells (Figure 8). Moreover, deficiency of miR-326 reversed knockdown of KCNQ1OT1-mediated limited capacity for c-Myc abundance in HL-60 and U937 cells (Figure 8). These findings revealed that KCNQ1OT1 functioned as a ceRNA for miR-326 to de-repress c-Myc expression in AML cells.

\section{Discussion}

LncRNAs have been indicated as important biomarkers for development, prognosis and treatment of AML [22]. In this study, we showed that KCNQ1OT1 expression was 

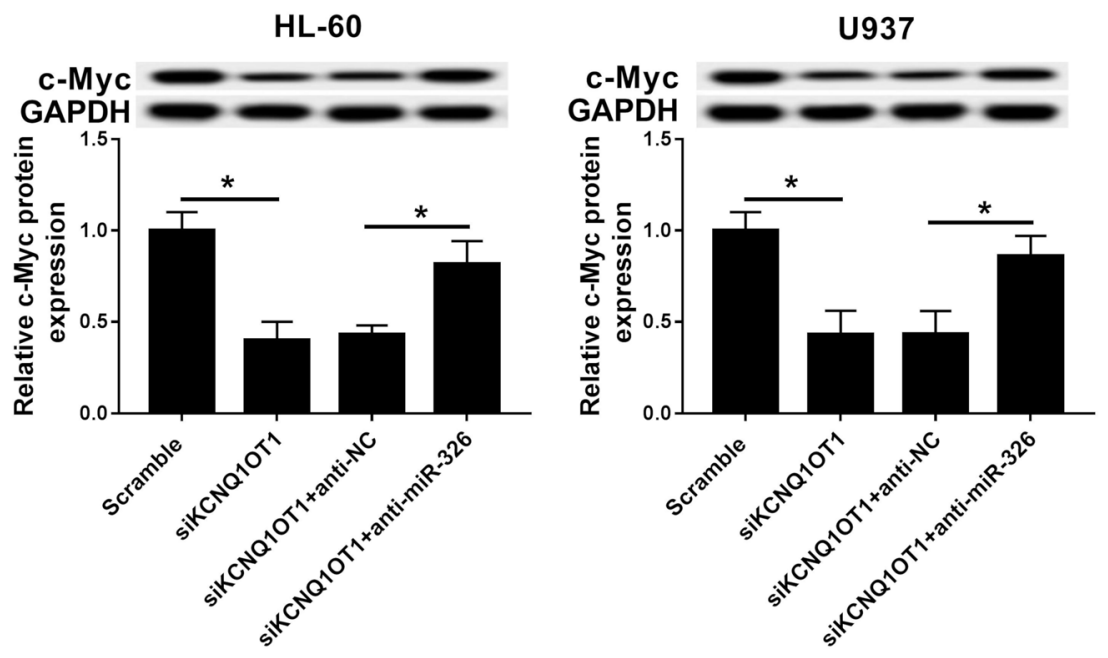

Figure 8. The abundance of c-Myc protein is regulated by KCNQ1OT1 and miR-326 in AML cells. The expression of c-Myc protein was measured in HL-60 and U937 cells transfected with scramble, siKCNQ1OT1, siKCNQ1OT1 and anti-NC or anti-miR-326 by western blot. ${ }^{*}$ p $<0.05$.

enhanced in AML samples compared with that in normal, which is also in agreement with previous study [12]. Moreover, high expression of KCNQ1OT1 was associated with lower survival in AML patients. These indicated that KCNQ1OT1 might predict poor outcomes of AML patients. However, the exact roles of KCNQ1OT1 in AML progression and its potential mechanism remain elusive. Here we first showed that KCNQ1OT1 regulated differentiation, proliferation and apoptosis by acting as a ceRNA for miR-326 to mediate c-Myc in AML.

PMA has been widely used to stimulate differentiation of human myeloid leukemia cell $[7,23]$. In this study, we also used PMA to induce differentiation of AML cells, which was confirmed by increase of CD14 and CD11b levels. Moreover, treatment with PMA decreased the expression of KCNQ1OT1 and silencing KCNQ1OT1 contributed to AML cell differentiation, which might be associated with cellular oxidant stress [24]. KCNQ1OT1 was reported as an oncogene by promoting tumor progression in human cancers [8-10, 25]. Similarly, our results also indicated the promoting role of KCNQ1OT1 in AML progression, revealed by which KCNQ1OT1 silence decreased proliferation by promoting apoptosis of AML cells, which is also in agreement with previous study [9]. In addition, the anti-proliferative effect of KCNQ1OT1 knockdown might be also caused by G2 cell cycle arrest [10], which needs to be further validated in future. These findings uncovered that KCNQ1OT1 silence might be a promising therapeutic target for AML through proliferation suppression and stimulating differentiation.

This study focused on the molecular mechanism that allows KCNQ1OT1 participating in AML process. Former works have indicated that KCNQ1OT1 could serve as important sponge of many miRNAs, including miR-145, miR-7-5p and miR-214 [10, 26, 27]. Here we first demonstrated the interaction between KCNQ1OT1 and miR-326 in AML cells, which was confirmed by luciferase activity, RIP and RNA pull-down assays. miR-326 expression was downregulated in AML samples, suggesting that miR-326 might be a suppressor in AML progression, which is also consistent with former work [17]. In this study, the expression of miR-326 was elevated in differentiated AML cells and its overexpression promoted cell differentiation, which is also in agreement with previous study suggesting that miR-326 promoted cell differentiation in pathogenesis of systemic lupus erythematosus by regulating Ets-1 [28]. Ets-1 is regarded as an important regulator of reactive oxygen species (ROS) [29], which further stimulated us to hypothesize that KNCQ1OT1 regulated cell differentiation by mediating oxidant stress. Moreover, miR-326 has been reported as a tumor suppressor by suppressing proliferation, invasion and activating apoptosis in hepatocellular carcinoma [16]. Our data revealed that miR-326 inhibited proliferation by promoting apoptosis in AML cells. Meanwhile, it might also be associated with G2 cell cycle arrest [30]. Notably, miR-326 knockdown reversed interference of KCNQ1OT1-mediated progression of AML, indicating KCNQ1OT1 as a sponge of miR-326.

Additionally, functional miRNAs are known to regulate target expressions in different conditions. Previous studies have confirmed multiple targets of miR-326, including kirsten rat sarcoma viral oncogene homolog (KRAS) and ETS-domain containing protein (ELK1) $[31,32]$. In the present study, c-Myc was identified as a functional target of miR-326 in AML cells by luciferase activity and RIP assays. Former effort revealed that c-Myc was highly expressed in AML [33]. Similarly, we also showed that c-Myc was elevated in AML samples. In addition, c-Myc was associ- 
ated with cell growth and differentiation in AML $[7,34]$. In this study, we found that miR-326 induced proliferation suppression and differentiation by targeting c-Myc in AML cells. Besides, the expression of c-Myc was reduced by knockdown of KCNQ1OT1, but this event was rescued by inhibition of miR-326, indicating that c-Myc was regulated by KCNQ1OT1 through competitively sponging miR-326. Recent research suggested that pre-clinical experiments are crucial for understanding the mechanism in AML development [35]. However, the limited outlay and experimental conditions led to the absence of in vivo data in the present study. Thus, the in vivo study is needed to be explored using an animal model in future.

In conclusion, the expressions of KCNQ1OT1 was increased in AML samples. Knockdown of KCNQ1OT1 repressed cell proliferation by promoting apoptosis and contributed to cell differentiation in AML cells, possibly by functioning as a ceRNA for miR-326 to mediate c-Myc, providing a new strategy for AML treatment.

\section{References}

[1] SHORT NJ, RYTTING ME, CORTES JE. Acute myeloid leukaemia. Lancet 2018; 392: 593-606. https://doi.org/10.1016/ S0140-6736(18)31041-9

[2] KHWAJA A, BJORKHOLM M, GALE RE, LEVINE RL, JORDAN CT et al. Acute myeloid leukaemia. Nat Rev Dis Primers 2016; 2: 16010. https://doi.org/10.1038/ nrdp.2016.10

[3] FENG Y, SHEN Y, CHEN H, WANG X, ZHANG R et al. Expression profile analysis of long non-coding RNA in acute myeloid leukemia by microarray and bioinformatics. Cancer Sci 2018; 109: 340-353. https://doi.org/10.1111/ cas. 13465

[4] YIN X, HUANG S, ZHU R, FAN F, SUN C et al. Identification of long non-coding RNA competing interactions and biological pathways associated with prognosis in pediatric and adolescent cytogenetically normal acute myeloid leukemia. Cancer Cell Int 2018; 18: 122. https://doi.org/10.1186/ s12935-018-0621-0

[5] ZHAO C, WANG S, ZHAO Y, DU F, WANG W et al. Long noncoding RNA NEAT1 modulates cell proliferation and apoptosis by regulating miR-23a-3p/SMC1A in acute myeloid leukemia. J Cell Physiol 2019; 234: 6161-6172. https:// doi.org/10.1002/jcp.27393

[6] SUN M, ZHENG Y, WANG L, ZHAO H, YANG S. Long noncoding RNA UCA1 promotes cell proliferation, migration and invasion of human leukemia cells via sponging miR-126. Eur Rev Med Pharmacol Sci 2018; 22: 2233-2245. https://doi.org/10.26355/eurrev_201804_14809

[7] CHEN L, WANG W, CAO L, LI Z, WANG X. Long NonCoding RNA CCAT1 Acts as a Competing Endogenous RNA to Regulate Cell Growth and Differentiation in Acute Myeloid Leukemia. Mol Cells 2016; 39: 330-336. https://doi. org/10.14348/molcells.2016.2308
[8] DONG Z, YANG P, QIU X, LIANG S, GUAN B et al. KCNQ1OT1 facilitates progression of non-small-cell lung carcinoma via modulating miRNA-27b-3p/HSP90AA1 axis. J Cell Physiol 2019; 234: 11304-11314. https://doi.org/10.1002/ jcp. 27788

[9] SUN H, LI Y, KONG H, DAI S, QIAN H. Dysregulation of KCNQ1OT1 promotes cholangiocarcinoma progression via miR-140-5p/SOX4 axis. Arch Biochem Biophys 2018; 658: 7-15. https://doi.org/10.1016/j.abb.2018.09.019

[10] FENG W, WANG C, LIANG C, YANG H, CHEN D et al. The Dysregulated Expression of KCNQ1OT1 and Its Interaction with Downstream Factors miR-145/CCNE2 in Breast Cancer Cells. Cell Physiol Biochem 2018; 49: 432-446. https:// doi.org/10.1159/000492978

[11] GAO X, GE J, LI W, ZHOU W, XU L. LncRNA KCNQ1OT1 promotes osteogenic differentiation to relieve osteolysis via Wnt/ $\beta$-catenin activation. Cell Biosci 2018; 8: 19. https://doi. org/10.1186/s13578-018-0216-4

[12] JIA ZW, LI Y, CUI GR, ZHAO HB, LI PY et al. [Expression and Clinical Significance of LncRNA KCNQ1OT1 in Patients with Acute Myeloid Leukemia]. Zhongguo Shi Yan Xue Ye Xue Za Zhi 2018; 26: 653-657. https://doi.org/10.7534/j. issn.1009-2137.2018.03.004

[13] TRINO S, LAMORTE D, CAIVANO A, LAURENZANA I, TAGLIAFERRI D et al. MicroRNAs as New Biomarkers for Diagnosis and Prognosis, and as Potential Therapeutic Targets in Acute Myeloid Leukemia. Int J Mol Sci 2018; 19. https://doi.org/10.3390/ijms19020460

[14] LIANG X, LI Z, MEN Q, LI Y, LI H et al. miR-326 functions as a tumor suppressor in human prostatic carcinoma by targeting Mucin1. Biomed Pharmacother 2018; 108: 574-583. https://doi.org/10.1016/j.biopha.2018.09.053

[15] WANG J, CAO L, WU J, WANG Q. Long non-coding RNA SNHG1 regulates NOB1 expression by sponging miR-326 and promotes tumorigenesis in osteosarcoma. Int J Oncol 2018; 52: 77-88. https://doi.org/10.3892/ijo.2017.4187

[16] HU S, RAN Y, CHEN W, ZHANG Y, XU Y. MicroRNA-326 inhibits cell proliferation and invasion, activating apoptosis in hepatocellular carcinoma by directly targeting LIM and SH3 protein 1. Oncol Rep 2017; 38: 1569-1578. https://doi. org/10.3892/or.2017.5810

[17] CAMMARATA G, AUGUGLIARO L, SALEMi D, AGUELI C, LA ROSA M et al. Differential expression of specific microRNA and their targets in acute myeloid leukemia. Am J Hematol 2010; 85: 331-339. https://doi.org/10.1002/ ajh. 21667

[18] BRONDFIELD S, UMESH S, CORELLA A, ZUBER J, RAPPAPORT AR et al. Direct and indirect targeting of MYC to treat acute myeloid leukemia. Cancer Chemother Pharmacol 2015; 76: 35-46. https://doi.org/10.1007/s00280-0152766-Z

[19] TIAN C, ZHENG G, ZHUANG H, LI X, HU D et al. MicroRNA-494 Activation Suppresses Bone Marrow Stromal CellMediated Drug Resistance in Acute Myeloid Leukemia Cells. J Cell Physiol 2017; 232: 1387-1395. https://doi.org/10.1002/ jcp. 25628 
[20] SAVVA CG, TOTOKOTSOPOULOS S, NICOLAOU KC, NEOPHYTOU CM, CONSTANTINOU AI. Selective activation of TNFR1 and NF- $\mathrm{KB}$ inhibition by a novel biyouyanagin analogue promotes apoptosis in acute leukemia cells. BMC Cancer 2016; 16: 279. https://doi.org/10.1186/s12885016-2310-5

[21] LIVAK KJ, SCHMITTGEN TD. Analysis of Relative Gene Expression Data Using Real-Time Quantitative PCR and the $2-\Delta \Delta$ C T Method. Methods 2001; 25: 402-408. https://doi. org/10.1006/meth.2001.1262

[22] GARZON R, VOLINIA S, PAPAIOANNOU D, NICOLET D, KOHLSCHMIDT J et al. Expression and prognostic impact of lncRNAs in acute myeloid leukemia. Proc Natl Acad Sci USA 2014; 111: 18679-18684. https://doi.org/10.1073/ pnas. 1422050112

[23] TIAN X, XIE G, DING F, ZHOU X. LPS-induced MMP-9 expression is mediated through the MAPKs-AP-1 dependent mechanism in BEAS-2B and U937 cells. ExpLung Res 2018; 44: 217-225. https://doi.org/10.1080/01902148.2018.1493551

[24] LAM CF, YEUNG HT, LAM YM, NG RK. Reactive oxygen species activate differentiation gene transcription of acute myeloid leukemia cells via the JNK/c-JUN signaling pathway. Leuk Res 2018; 68: 112-119. https://doi.org/10.1016/j. leukres.2018.03.012

[25] LI C, MIAO R, ZHANG J, QU K, LIU C. Long non-coding RNA KCNQ1OT1 mediates the growth of hepatocellular carcinoma by functioning as a competing endogenous RNA of miR-504. Int J Oncol 2018; 52: 1603-1612. https://doi. org/10.3892/ijo.2018.4313

[26] HU H, YANG L, LI L, ZENG C. Long non-coding RNA KCNQ1OT1 modulates oxaliplatin resistance in hepatocellular carcinoma through miR-7-5p/ ABCC1 axis. Biochem Biophys Res Commun 2018; 503: 2400-2406. https://doi. org/10.1016/j.bbrc.2018.06.168

[27] JIN X, JIN H, SHI Y, GUO Y, ZHANG H. Long Non-Coding RNA KCNQ1OT1 Promotes Cataractogenesis via miR-214 and Activation of the Caspase-1 Pathway. Cell Physiol Biochem 2017; 42: 295-305. https://doi.org/10.1159/000477330
[28] JIN L, FANG X, DAI C, XIANG N, TAO J et al. The potential role of Ets-1 and miR-326 in CD19B cells in the pathogenesis of patients with systemic lupus erythematosus. Clin Rheumatol 2019; 38: 1031-1038. https://doi.org/10.1007/s10067018-4371-0

[29] NI W, ZHAN Y, HE H, MAYNARD E, BAISCHI JA et al. Ets1 is a critical transcriptional regulator of reactive oxygen species and p47(phox) gene expression in response to angiotensin II. Circ Res 2007; 101: 985-994. https://doi.org/10.1161/ CIRCRESAHA.107.152439

[30] JI S, ZHANG B, KONG Y, MA F, HUA Y. miR-326 Inhibits Gastric Cancer Cell Growth Through Downregulating NOB1. Oncol Res 2017; 25: 853-861. https://doi.org/10.3727 /096504016X14759582767486

[31] KANG K, ZHANG J, ZHANG X, CHEN Z. MicroRNA-326 inhibits melanoma progression by targeting KRAS and suppressing the AKT and ERK signalling pathways. Oncol Rep 2018; 39: 401-410. https://doi.org/10.3892/or.2017.6074

[32] ZHANG P, KONG F, DENG X, YU Y, HOU C et al. MicroRNA-326 suppresses the proliferation, migration and invasion of cervical cancer cells by targeting ELK1. Oncol Lett 2017; 13: 2949-2956. https://doi.org/10.3892/ol.2017.5852

[33] GE Z, GUO X, LI J, HARTMAN M, KAWASAWA Y et al. Clinical significance of high c-MYC and low MYCBP2 expression and their association with Ikaros dysfunction in adult acute lymphoblastic leukemia. Oncotarget 2015; 6: 42300-42311. https://doi.org/10.18632/oncotarget.5982

[34] MANIWA Y, KASUKABE T, KUMAKURA S. Vitamin K2 and cotylenin A synergistically induce monocytic differentiation and growth arrest along with the suppression of c-MYC expression and induction of cyclin G2 expression in human leukemia HL-60 cells. Int J Oncol 2015; 47: 473-480. https:// doi.org/10.3892/ijo.2015.3028

[35] GELEBART P, POPA M, MCCORMACK E. Xenograft Models of Primary Acute Myeloid Leukemia for the Development of Imaging Strategies and Evaluation of Novel Targeted Therapies. Curr Pharm Biotechnol 2016; 17: 42-51. https://doi.or g/10.2174/1389201016666150817095703 